\title{
Research on Simulation of Distribution Network Engineering Scene Based on 3D GIS Technology
}

\author{
Qiong Wang (D) and Yan Li \\ State Grid Jiangsu Economic Research Institute, Nanjing 210000, China \\ Correspondence should be addressed to Qiong Wang; joanwong2021@163.com
}

Received 9 December 2021; Revised 28 December 2021; Accepted 30 December 2021; Published 31 January 2022

Academic Editor: Xin Ning

Copyright (c) 2022 Qiong Wang and Yan Li. This is an open access article distributed under the Creative Commons Attribution License, which permits unrestricted use, distribution, and reproduction in any medium, provided the original work is properly cited.

\begin{abstract}
With the rapid development of the power system and the increasing complexity of the power grid structure, users' demand for information-based management of the power distribution network is growing, and the need to improve various power production service standards is becoming increasingly urgent. Geographic information systems (GIS) are a natural combination of geographic and data attributes that are particularly well suited to the management of urban pipeline systems such as distribution and telecommunication networks. This paper discusses the current application characteristics and challenges of 3D GIS in the power system. A 3D graphic analysis model of the power grid and its realization method are proposed using 2D GIS and scene simulation technology combined with object-oriented design method. The problems that exist in current urban distribution facility management are solved in this paper through the effective integration of information construction and distribution facility management, and the goal of refined management is achieved on this basis. More and more complex 3D spatial data can be processed more realistically and intuitively with 3D GIS.
\end{abstract}

\section{Introduction}

Transmission line engineering is a large-scale undertaking. Regional spatial information is in high demand for everything from power grid planning, design, and construction to operation, management, and maintenance $[1,2]$. A geographic information system (GIS) is a data storage, analysis, and presentation system for spatial and nonspatial data [2]. It is part of a larger system for gathering, storing, converting, extracting, and expressing related geographic phenomena in the real world [3]. It, as well as a combination of tools with intrinsic relevance, can assist people in solving a wide range of complex geographical problems. In recent years, 3D GIS technology, supported by spatial visualization technology [4] and spatial database technology, has developed vigorously, making breakthrough progress in large-scale terrain display, spatial data management and analysis, etc., which provides necessary conditions for the research of a new generation of visual distribution network management system [5].

With the rapid development of power systems and the increasing complexity of power grid structures, users' demands to manage the operation of power distribution networks through information are increasing, and the need to improve various power production service standards is becoming increasingly urgent [6]. The three-dimensional distribution network system consists of a three-dimensional digital terrain model, high-resolution remote sensing image data, three-dimensional power facilities and equipment model, three-dimensional visual simulation of power grid equipment, and a power grid construction area and allows users to browse, roam, position, and perform other operations in the three-dimensional scene interactively [7, 8]. The transmission line is long in distance, and the geographical conditions of the passing area are complex, and it crosses with many power lines and communication lines [9]. In order to improve the management level of the power industry, the power industry has to strengthen the management of power distribution network through information technology [10]. Based on this, this paper introduces the application characteristics and difficulties of 3D GIS in power system. By using two-dimensional GIS and scene simulation technology, combined with object-oriented design method, 
this paper puts forward a method to build a threedimensional graphic analysis model of power grid and its implementation method.

Power distribution network facilities include power equipment, auxiliary equipment, and their operating environment. The management of distribution network facilities requires integrated management of power equipment, auxiliary equipment, and their operating environment [11]. However, the current information system only describes the power equipment and does not include the auxiliary facilities of power equipment, and the managers cannot grasp the environmental information and overall situation of power equipment [12]. 3D GIS can display relevant data information in a 3D way, such as the positional relationship between iron tower and conductor, the electrical gap between conductor and ground wire, and the distance between conductor to ground and crossing distance [13]. $3 \mathrm{D}$ GIS can deal with more and more complex 3D spatial data more truly and intuitively [14]. Through the effective integration of information construction and distribution network facilities management, this paper solves the problems existing in the current urban distribution network facilities management and realizes the goal of fine management on this basis. 3D GIS has obvious advantages over 2D GIS in $3 \mathrm{D}$ scene visualization and real-time roaming. The analysis, evaluation, and decision-making of problems also get rid of the limitation that 2D GIS is only based on plane problem-solving.

\section{Related Work}

Distribution network is the most important part of the foundation of power supply, which directly supplies power to end users. How to ensure the maximum power supply and provide better services for users to improve the overall efficiency of the power industry is a problem that electric power enterprises must face and solve at present. Literature [15] pointed out that the management status of distribution network facilities has a direct relationship with the construction of 3D distribution network system, and a key factor for the success of the construction of 3D GIS distribution network system lies in the accurate positioning and description of distribution network facilities. Literature [16] mainly discusses the management methods and processing methods of multidimensional spatial information. Literature [17] will combine with and use its modeling function to create a three-dimensional distribution network system to realize the related functions required by users. Literature [18] comprehensively analyzes the respective characteristics and advantages of 3D modeling and based secondary development software and builds a 3D power distribution network model by taking advantage of the simplicity and quickness of the software in 3D modeling. Literature [19] takes the application practice of 3D GIS in a power grid company as the research background to solve the key problems in the field management and implementation of power distribution facilities. Literature [20] discusses the integration of threedimensional information technology and distribution network management from the process construction of infor-

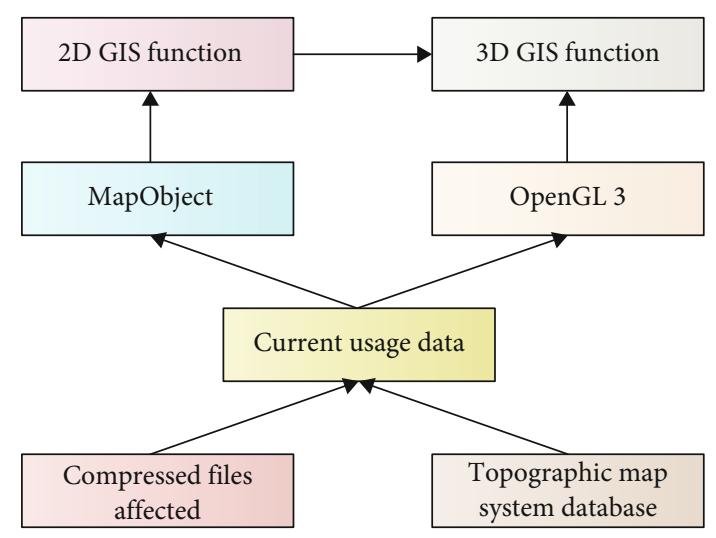

Figure 1: The structural design of the system.

mation system to solve the problems in distribution network management. In literature [21], by using 3D scene simulation technology and object-oriented design method, a method combining 2D GIS with scene simulation technology is proposed. The literature [22] examines the application mode of a 3D GIS system in power grid design, operation, and maintenance from multiple perspectives of overhead transmission lines and substation design and construction. Based on current research findings, this paper optimizes product architecture design and improves the visualization effect of power grid data and proposes a new generation of distribution network engineering scene simulation technology in order to shorten the R\&D cycle, maintain product technological advancement, and increase market competitiveness.

\section{Application Characteristics and Difficulties of 3D GIS Technology in Power System}

Electric power distribution system is a comprehensive management system which combines computer programming language, real-time communication technology, electronic information technology, and other related technologies and uses intelligent operation means to realize the functions of power supply range control, power outage range control, and detection of electric power distribution network. From two-dimensional map, sand table, animation to virtual scene simulation, it is an inevitable result that accords with the deepening of people's understanding and the development trend of technology. The greatest advantage of 3D GIS system is that it can truly reproduce the geographic information in the real environment. In the daily management of power distribution system, related equipment is closely related to its geographical space environment, that is, it is inseparable from geographical coordinates. Different from the equipment of transmission network, the equipment of distribution network is generally installed on multibase towers, which are scattered in distribution, with many points and wide areas, and the relationship between them is complicated [23]. Using 3D GIS technology can realize the realistic 3D model generation function and real-time roaming function. The structural design of the system is shown in Figure 1. 


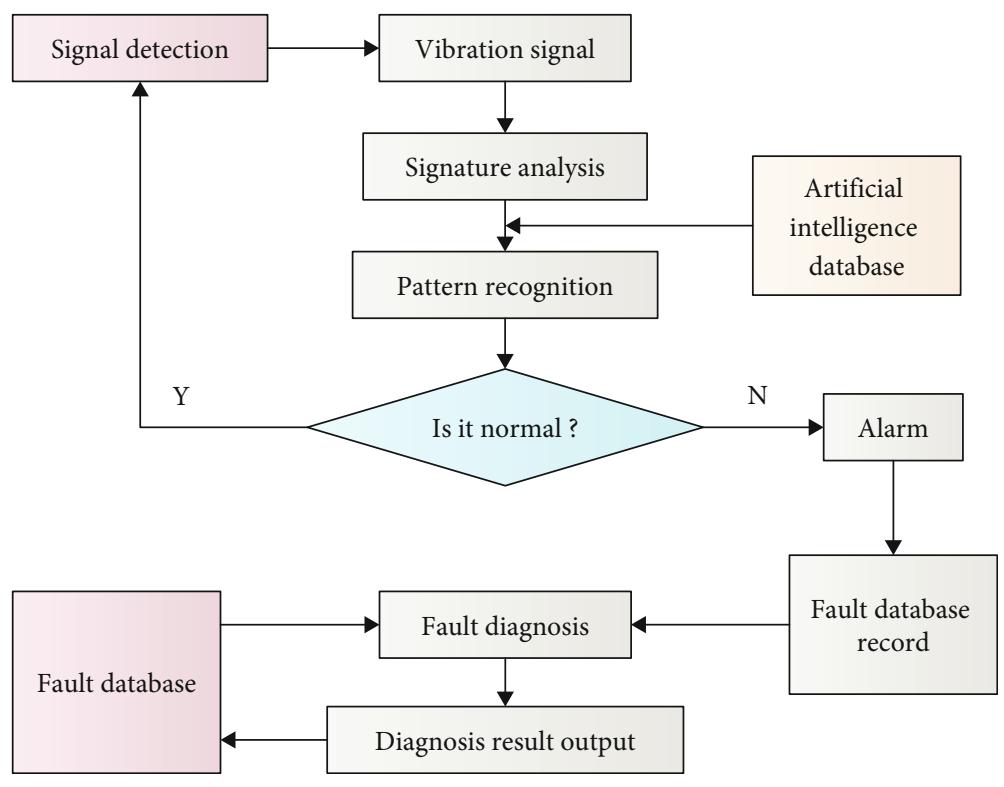

Figure 2: Fault diagnosis system.

There are still technical limitations in 3D GIS, such as 3D data acquisition, processing and storage of large amounts of data, 3D visualization, and 3D spatial analysis, which prevent it from meeting the needs of large-scale commercial applications while remaining cost-effective. If $3 \mathrm{D}$ GIS is used in its entirety, the system will inevitably cost a lot of money to build. Users do not need to pursue high performance blindly when 2D GIS can meet their needs. The transmission network's connection mode is mostly simple mesh, while the distribution network is blessed. As a result, the distribution system's topology is far more complicated. We should not only be familiar with the geographical location of the system equipment during routine management but also with its complex network structure and topological relationships, in order to ensure the normal operation and management of the distribution network system's various functions.

\section{Model Construction of Power Distribution Network System}

The GIS system of electric power distribution network is an advanced information management system, which is based on computer network technology, GIS technology, and relational database technology and established by using computer programming language and integrates the operation, monitoring, maintenance, and management of distribution equipment. The system's goal is to create a 3D scene using an open information technology framework system, with digital earth as the carrier, high-resolution image data, digital elevation models, and high-precision $3 \mathrm{D}$ power grid models as the foundation, and 3D GIS as the platform, and to integrate basic geographic information with image, power grid, power supply, substation, and related power thematic data using data organization, spatial analysis, and calculation. Network of electric power distribution for the manage- ment departments of electric power enterprises, GIS can provide complete details, more intuitive and accurate information, and decision-making services [10]. It provides technical support for visual display, storage, retrieval, and intelligent analysis of spatial coordinates and other related attribute information of power distribution equipment and realizes the automation of database management system, relying on the powerful functions of GIS in spatial coordinate representation and spatial analysis. The system adopts today's advanced service-oriented SOA architecture, shares attribute data with the power grid production management system by constructing a power grid information service platform based on three-dimensional spatial geographic information, ensures data integrity, and develops and integrates various power grid planning functional modules.

Three-dimensional virtual terrain environment is the foundation and framework of three-dimensional geographic information system. First of all, it is necessary to solve the storage problem of massive terrain data needed to build the scene. GIS is widely used in normal operation, troubleshooting, restoration of regional power supply, analysis and design of distribution system, etc. Different from the transmission network, the number of distribution equipment is huge and easy to change. One end of the system is connected to the power supply equipment and network, and the other end is directly connected to the vast number of user equipment, which is numerous in number, different in nature, and often changing. Figure 2 shows the framework of fault diagnosis system for power intranet.

In the transmission line GIS system, a complete line can span tens or even hundreds of kilometers, necessitating a large-scale digital ground model. Furthermore, the complex structure of the entire three-dimensional scene is caused by numerous surface feature models such as rivers, roads, residential areas, and tens of millions of transmission equipment models, and it is impossible to load all data into the 


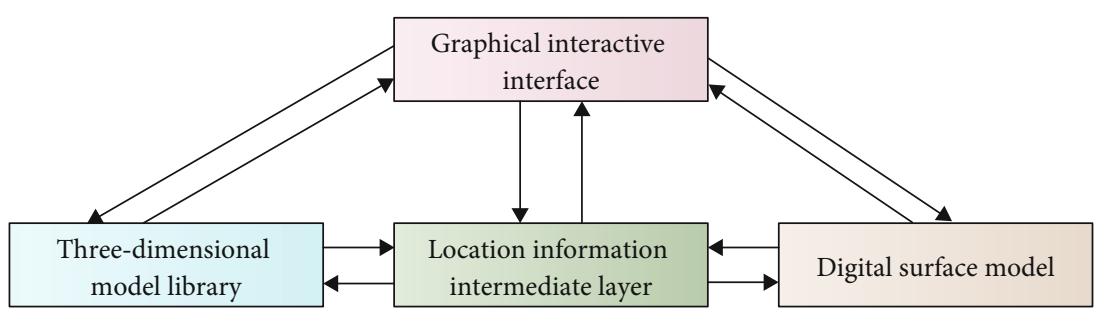

FIGURE 3: Three-tier system structure.

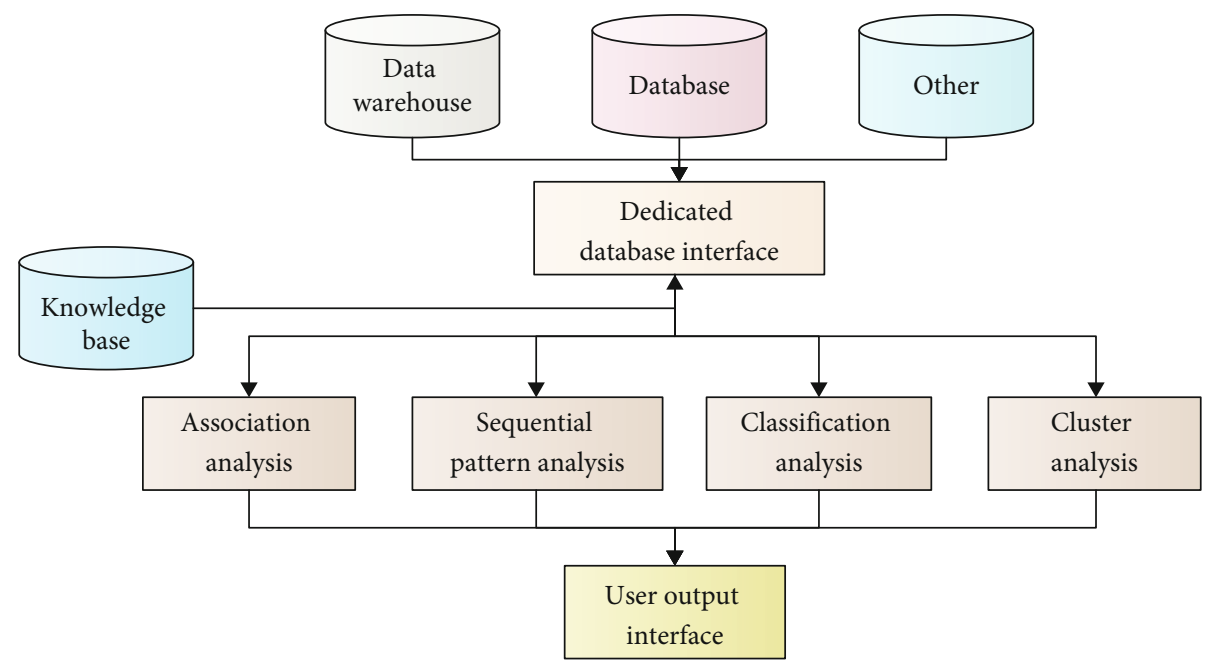

FIgURE 4: Power grid data mining based on data integration.

main memory or even the virtual memory at once. These technologies enable the creation of more realistic 3D surface models and various $3 \mathrm{D}$ object models, allowing GIS systems to progress from $2 \mathrm{D}$ to $3 \mathrm{D}$. Three-dimensional digital terrain model, high-resolution remote sensing image data, and three-dimensional simulation scenes of three-dimensional power facilities and equipment models are all part of the three-dimensional distribution network system. It can simulate three-dimensional visualization of power grid equipment and construction areas, and it allows users to browse, roam, position, and perform other operations in three-dimensional scenes interactively. According to the technical characteristics of 3D GIS and the basic requirements of line management, a three-tier architecture of the system is proposed, as shown in Figure 3.

In GIS of power distribution network, using relational database technology and computer graphics processing technology, it is possible to display the poultry risks and other related information of all related distribution equipment on the corresponding geographic map. Using related functions, the system can also comprehensively manage the single-line diagram, structure diagram, and entered electronic documents and materials of the power distribution network, which greatly promotes the visualization and automation of the power distribution network. Field construction has taken a significant step toward transforming power facilities to meet construction standards. The data of distribution network facilities is transformed from entities to information descriptions through the process of data col- lection, sorting, and entry, and the transformed facilities are displayed in the system [24]. The GIS system of the electric power distribution network uses data capture database technology to link spatial and attribute information of an electric power device, allowing equipment maintenance personnel to understand the device's operation in real time. As a result, if a device fails, a replacement can be quickly installed, eliminating the failure and greatly increasing dispatching efficiency. Its primary purpose is to query spatial data and conduct comprehensive analyses.

In order to successfully carry out the data collection of 3D distribution network, it is necessary to put forward the matters needing attention, collection contents and steps of the collection staff to ensure the quality of data engineering implementation delivery results, and also to formulate relevant implementation standards and action plans. The implementation standard is formulated according to the requirements of the three-dimensional distribution network system and the contents involved in the production and operation management. The architecture of power grid data mining based on data integration technology is shown in Figure 4.

The objective function of the nonlinear optimization problem with the minimum total generation cost of distribution network as the objective number is expressed as

$$
C\left(P_{G}\right)=\sum_{i=1}^{n} C_{i}\left(P_{G, i}\right) .
$$




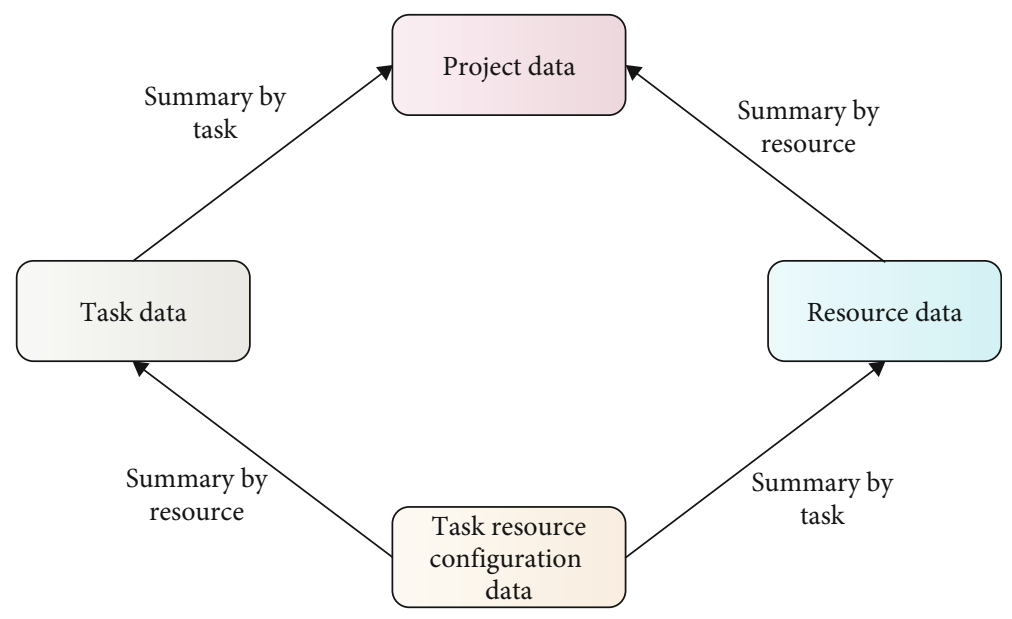

FIGURE 5: Manage project data relationships.

Meet the following power balance conditions:

$$
P_{d}-\sum_{i=1}^{n} P_{G, i}=0 .
$$

$P_{G}=\left|P_{G, 1}, P_{G, 2}, \cdots, P_{G, n}\right|$ is the output vector composed of the output of each generator, $P_{G, i}$ is the active output of the $i$-th generator, $C_{i}\left(P_{G, i}\right)$ is the cost function of the $i$-th generator, and $P_{d}$ is the total load of the power grid.

There are still technical limitations in 3D GIS, such as 3D data acquisition, processing and storage of large amounts of data, 3D visualization, and 3D spatial analysis, which prevent it from meeting the needs of large-scale commercial applications at a reasonable cost. If 3D GIS is used entirely, the cost of system development will inevitably be high. Users do not have to pursue high performance blindly when 2D GIS can meet their needs. Perfect text editing and graphic editing functions should be included in the system function supplement when building a GIS to make data maintenance more convenient. The management data relationship model is shown in Figure 5.

If it is necessary to highlight the route and connection relationship of the line, the location relationship between the station and the equipment, the method of layered blanking can be adopted to hide temporarily unnecessary layers, or the gray scale can be adjusted. At present, there is no mature solution of $3 \mathrm{D}$ spatial database. The $3 \mathrm{D}$ model data in 3D GIS system and its orientation information and related attribute information in 3D scene are often stored in different ways. The former is generally in file format and cannot be shared, while the latter can be placed in a general relational database. After the introduction of GIS technology, the power distribution system also uses the advantages of GIS visual management to abstract all kinds of data representing spatial things into three categories: point, line, and surface. Among them, points are used as basic units in the map, such as towers in the distribution network and all independent symbols that do not scale with the scale. The line represents the line-like objects in the distribution network, which are distinguished by different colors, shapes, and thicknesses. The surface is represented as a closed polygon, such as a planar object such as a power distribution station house in the distribution network.

\section{Optimization of On-Site Power Facility Management}

With the continuous expansion of power grid scale, in order to add, delete, or modify related data timely and accurately, it is required that the system can respond in time [25]. When a permanent fault occurs in the subscriber line or its operation mode changes, the system can efficiently guarantee the reconfiguration of its topological structure. After the completion of the power grid, in the face of the long power network that has been built, how to manage it effectively to ensure the normal operation of the power grid and the normal transmission of power also becomes more important. Because of a power outage, it will not only bring great economic losses to power grid enterprises but also seriously affect power users and the whole society.

By using 3D GIS technology, at the stage of optimizing route selection in preliminary design, the corresponding professional analysis functions are developed on the basis of 3D GIS system, providing various perspectives of $2 \mathrm{D}$ map, plane section map, and $3 \mathrm{D}$ map and moving the $3 \mathrm{D}$ real scene around the field line indoors so that designers can fully consider the influencing factors of terrain, social environment, and ecological environment and optimize the design. In the aspect of short-term dispatching, because of the randomness and uncontrollability of electric power, it will increase the rotating reserve capacity of the system and change the start-stop strategy of conventional units after it is connected to the network, which may lead to the increase of the operating cost of the power system. The algorithm evolution curve is shown in Figure 6.

At present, there is no mature solution of $3 \mathrm{D}$ spatial database. The 3D model data in 3D GIS system and its orientation information and related attribute information in $3 \mathrm{D}$ scene are often stored in different ways. Threedimensional GIS system can provide all-digital threedimensional visual management of line corridor assets in 


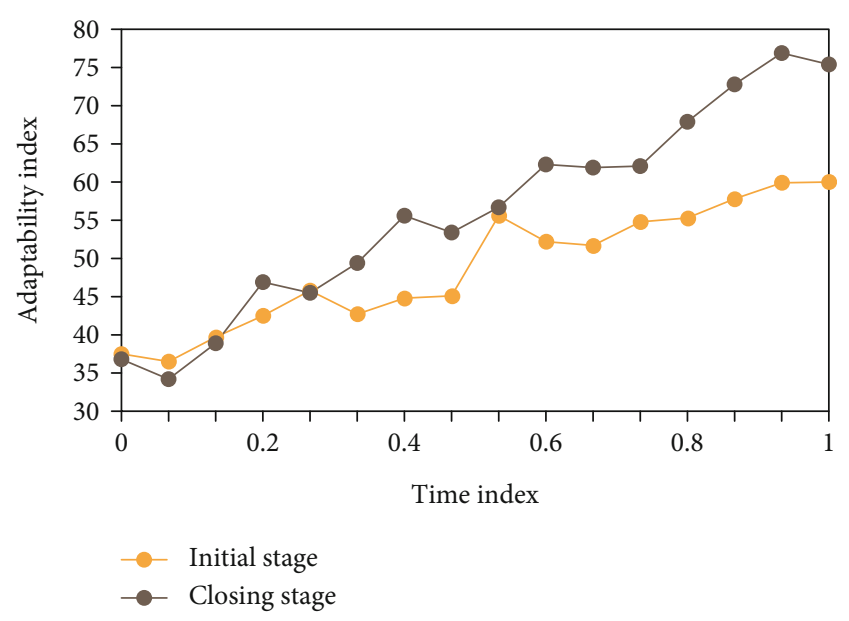

FIGURE 6: Algorithm evolution curve.

an intuitive way in combination with the needs of power grid operation and maintenance. Compared with the traditional drawings, reports, and electronic files, the result transfer of newly designed lines or substations can provide a complete data management and distribution service system in various unique forms such as full digitization, system modularization, and three-dimensional visualization. Considering that the change of the model in the scene is mainly the change of position, the corresponding 3D model prototype is established for each type of equipment or features, and the prototype library is formed. Then, when the system is running, an instance is established for each device to be displayed in the scene or other model individuals to participate in interactive operation. This example gets the corresponding orientation information and equipment attribute information from relational database and records one or more model indexes pointing to the $3 \mathrm{D}$ model prototype database at the same time.

The modern database system specification design method focuses primarily on the design of physical and logical databases. After determining the logical structure, the physical database's design, implementation details, and storage structure are determined. The goal of logical database design is to create global and local logical structures that meet the needs and characteristics of specific database management systems. After improving the data, the 3D GIS distribution network management system displayed the distribution network facilities in a refined and integrated manner, achieving an effect that previous information systems were unable to achieve and resolving the problems of difficult positioning and sloppy data in the management of distribution network facilities. Refer to the adopted characteristic data of conventional units, and bear the peak load or standby load as needed. The cost optimization curve is shown in Figure 7.

Data integrity includes whether the geometric description of power grid resource data and spatial geographic data is complete, whether the data stratification is correct, and whether there are omissions or duplicates. On the basis of the same precision and accuracy, the degree of completeness that data can achieve in a specific spatial range and type. The

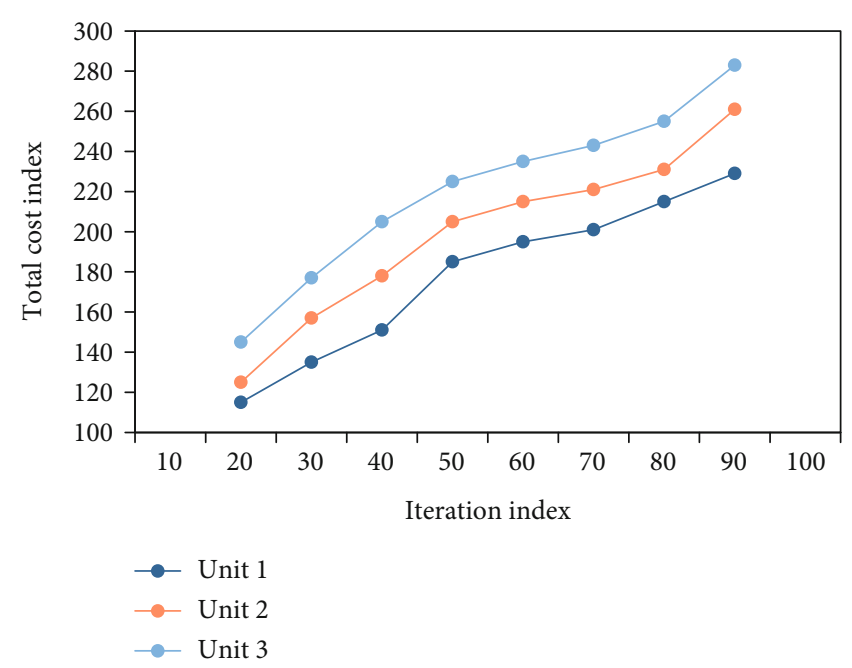

FIgURe 7: Cost optimization curve.

field real-life environment is simulated in the computer during the substation design process using three-dimensional technology, which can be used for comparison and selection of substation site schemes as well as three-dimensional display of design results. Designers can compare and select large schemes of substation and power station sites in 2D and 3D mode, as well as constraints such as planning data, to improve the feasibility and accuracy of substation and power station site selection. The majority of power system optimization problems necessitate multiobjective optimization. Unlike single-objective optimization, which only requires extreme value, the relationship between multiple objectives in multiobjective optimization problems is contradictory. When one goal is at its best, another may be in the worst possible state. As a result, we must make some compromises when dealing with multiobjective optimization problems, observing the health assessment and judging the result of defect extraction. The deviation curve of fault characteristics is shown in Figure 8.

Based on the topological potential of cyberphysical system network, the potential distribution and mutual influence of network elements in the network can be described mathematically. Therefore, the identification method of key network elements based on topological potential can be established, and the backup protection strategy of nodes with high topological potential can be put forward to improve the vulnerability of cyberphysical systems from the point of view of node protection. The power flow optimization of AC/DC hybrid distribution network is a nonlinear and discrete optimization problem mathematically. Intelligent algorithm is an effective method to solve this kind of problem, and it has better applicability than analytical method. Figure 9 shows the optimization results of total unit cost under different risk factors.

In the process of power transmission in the distribution network system, due to the existence of impedance, transmission loss caused by impedance inevitably exists in the distribution network system. In the traditional AC distribution network, as a passive network, it is difficult to adjust the 


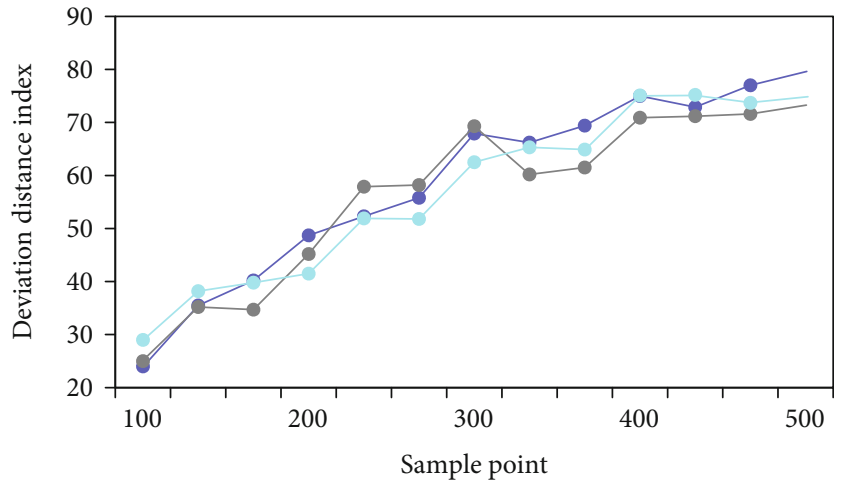

- Degenerate state

- Normal state Fault state

FIgURE 8: Fault characteristic deviation curve.

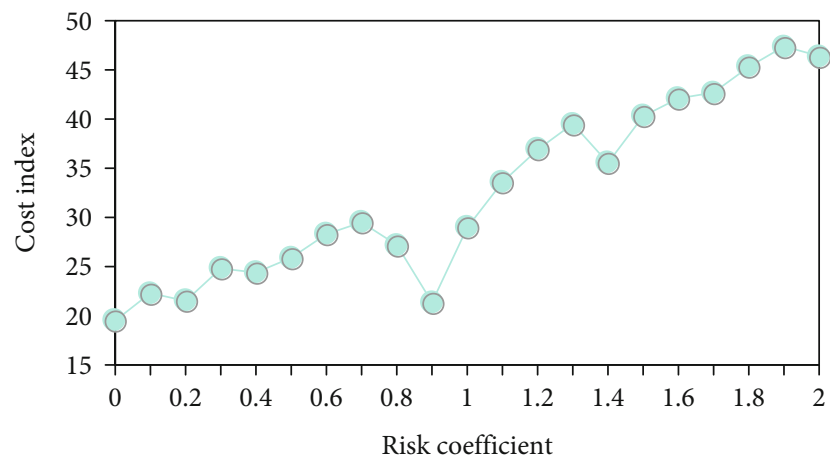

FIgURE 9: Optimization results of unit total cost under different risk factors.

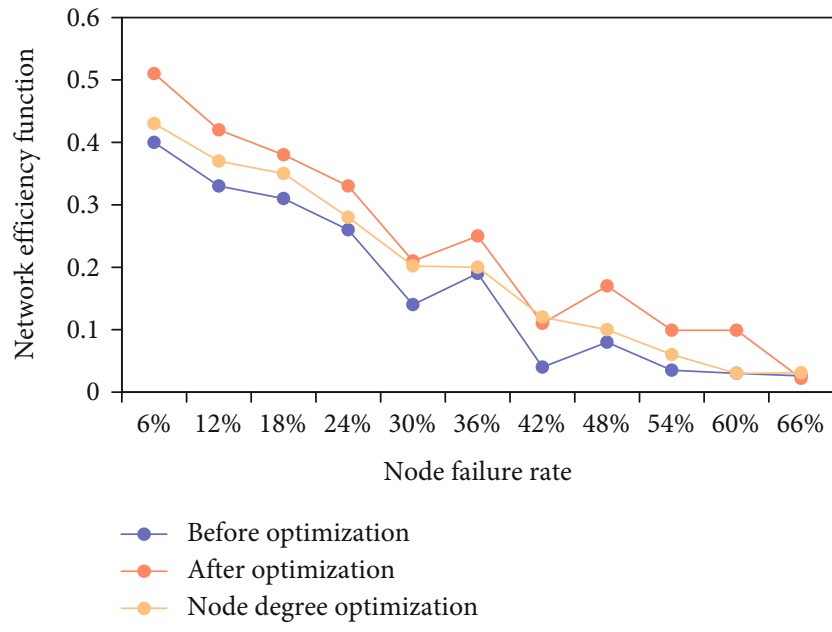

FIgURE 10: Comparison of network efficiency functions with different optimization strategies.

system loss. However, with the development of power electronics technology and the large-scale distributed power supply connected to the distribution network, the system loss has certain adjustability. The primary goal of database

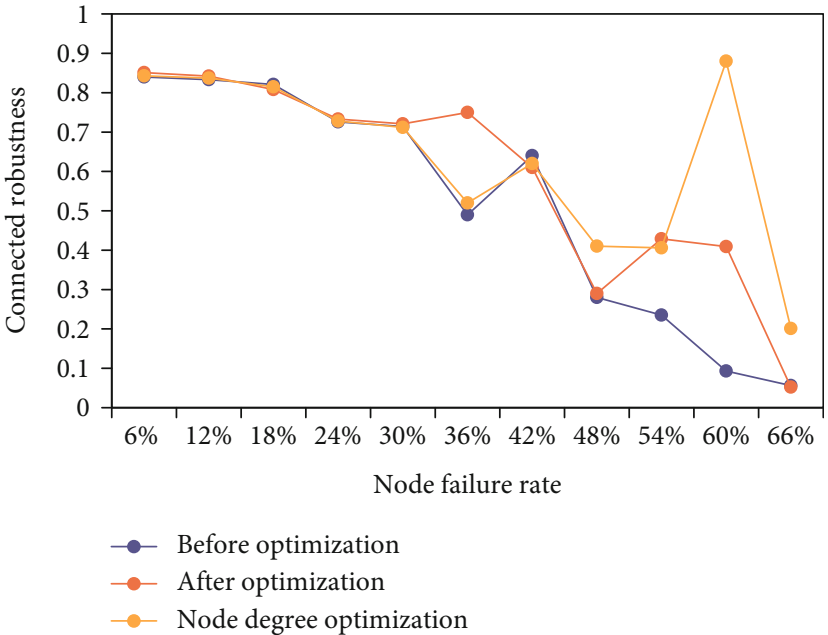

FIGURE 11: Comparison of connectivity and robustness of different optimization strategies.

design is to grasp and analyze the actual needs of power distribution network users, including information and operation, and then determine the software configuration scheme and overall research and development platform required for system development. The power grid is a complicated transmission and distribution hybrid power grid with a wide range of power equipment and construction types, such as outdoor, indoor, and underground substations. To demonstrate the effectiveness of the proposed algorithm, the network performance before and after optimization is compared using connectivity and network efficiency functions. In the case of large-scale fault, the method of node uncertain failure is used to simulate the distribution network project scenario. Comparison of network efficiency functions of different optimization strategies under uncertain failure of nodes is shown in Figure 10.

The reliability of distribution network project scenario can be expressed by connected robust function. Similarly, the simulation method of uncertain failure of nodes is used. Compared with node edge algorithm, topology optimization based on 3D GIS can maintain higher network reliability, as shown in Figure 11.

It can be seen that the distribution network engineering scene simulation method based on 3D GIS proposed in this paper can solve the network planning problems with different heterogeneous backgrounds and has good application potential. According to the operation rules of power grid, the electric power distribution system uses GIS technology to comprehensively manage the engineering equipment and production technology in relevant geographical areas and classifies its internal application data according to relevant rules by so-called "structured organization." For 3D model visualization and user interaction interface development, the author chooses Java language, which has good cross-platform characteristics and can be transplanted to the current mainstream WEB-based applications without much work. The electric power distribution system can display the distribution map of distribution lines in the form of electronic map by using the visualization technology of GIS 
system and unify the spatial and attribute information in the system by using the established database, thus realizing the more scientific management of the distribution system.

\section{Conclusions}

With the progress and development of science and technology, 3D GIS technology is developing at an unprecedented speed and plays an important role in the design, operation, and maintenance of power grid. Real life is a world full of three-dimensional objects. Only a three-dimensional GIS system can make people know and understand geography and equipment information more intuitively and vividly, thus improving management level and work efficiency. According to the technical characteristics of 3D GIS and the requirements of transmission line management, this paper puts forward a simulation method of distribution network engineering scene based on 3D GIS technology by making rational use of 3D model construction and design methods. The simulation method of distribution network engineering scene based on 3D GIS proposed in this paper can solve the network planning problems with different heterogeneous backgrounds and has good application potential. After improving the data, the 3D GIS distribution network management system showed the distribution network facilities in a refined and integrated way, which realized the effect that the previous information systems could not achieve and solved the problems of difficult positioning and rough information in the management of distribution network facilities.

After that construction of the three-dimensional distribution network facilities management system is complete, how to establish a set of management mechanism to dynamically manage the change of distribution network facilities, so as to keep the system consistent with the site, is the place that this article failed to deeply explore. Three-dimensional GIS system platform displays intuitive geographic data; combined with professional analysis tools and system functions, it will gradually realize the digitalization and intelligence of power grid construction.

\section{Data Availability}

The data used to support the findings of this study are included within the article.

\section{Conflicts of Interest}

All the authors do not have any possible conflicts of interest.

\section{Acknowledgments}

This paper is funded by the consulting project "Research on 3D digital handover and visualization application of distribution network based on power grid resource business center of State Grid Jiangsu Economic Research Institute" of State Grid Jiangsu Economic Research Institute (B710K0216LQD).

\section{References}

[1] Y. Nan and W. Kaisheng, "Power grid video monitoring system based on 3D GIS," China Electric Power, vol. 45, no. 4, p. 6, 2012.

[2] A. Aly, S. S. Jensen, and A. B. Pedersen, "Solar power potential of Tanzania: identifying CSP and PV hot spots through a GIS multicriteria decision making analysis," Renewable Energy, vol. 113, no. 12, pp. 159-175, 2017.

[3] Z. Yadi, W. Hongjie, Z. Hong, and D. Zhang, "Design and application research of power grid data asset visualization system based on 3D GIS platform," Electrical Measurement \& Instrumentation, vol. 55, no. 7, p. 6, 2018.

[4] M. Zhao, Q. Liu, A. Jha, R. Deng, T. Yao, and A. MahadevanJansen, "VoxelEmbed: 3D instance segmentation and tracking with voxel embedding based deep learning," 2021, https://arxiv .org/abs/2106.11480.

[5] K. Liu, M. M. Khan, A. Rana, and D. Fei, "Power flow control at the subnetwork-level in microgrids," Journal of Power Electronics, vol. 18, no. 2, pp. 588-603, 2018.

[6] D. Szalaj, L. Wise, S. Rodríguez-Climent et al., "A GIS-based framework for addressing conflicting objectives in the context of an ecosystem approach to fisheries management-a case study of the Portuguese sardine fishery," ICES Journal of Marine Science, vol. 75, no. 6, pp. 2070-2087, 2018.

[7] G. Mazzanti, G. Stomeo, and S. Mancini, "State of the art in insulation of gas insulated substations: main issues, achievements, and trends," IEEE Electrical Insulation Magazine, vol. 32, no. 5, pp. 18-31, 2016.

[8] M. Giamalaki and T. Tsoutsos, "Sustainable siting of solar power installations in Mediterranean using a GIS/AHP approach," Renewable Energy, vol. 141, no. 10, pp. 64-75, 2019.

[9] G. Garegnani, S. Sacchelli, J. Balest, and P. Zambelli, "GISbased approach for assessing the energy potential and the financial feasibility of run-off-river hydro-power in Alpine valleys," Applied Energy, vol. 216, no. 4, pp. 709-723, 2018.

[10] Z. Hao, "Research and implementation of GIS-based threedimensional auxiliary design system for transmission lines," Value Engineering, vol. 38, no. 12, pp. 167-169, 2019.

[11] W. Yang Ke, Y. X. Faxian, and Y. Liu, "Research on threedimensional GIS technology in the visual display platform of transmission lines," Science and Technology Innovation Herald, vol. 14, no. 1, p. 3, 2017.

[12] M. Hongcheng, Q. Jianguo, and Y. Ge, "Development and implementation of WebGIS for 3D power grid based on cesium," Surveying and Spatial Geographic Information, vol. 41, no. 11 , p. 4, 2018.

[13] Z. Akhtar and M. A. Saqib, "Microgrids formed by renewable energy integration into power grids pose electrical protection challenges," Renewable Energy, vol. 99, no. 12, pp. 148-157, 2016.

[14] H. Z. al Garni and A. Awasthi, "Solar PV power plant site selection using a GIS-AHP based approach with application in Saudi Arabia," Applied Energy, vol. 206, no. 9, pp. 12251240, 2017.

[15] S. Werner and J. Lunden, "Smart load tracking and reporting for real-time metering in electric power grids," IEEE Transactions on Smart Grid, vol. 7, no. 3, pp. 1723-1731, 2016.

[16] S. Soltan, A. Loh, and G. Zussman, "A learning-based method for generating synthetic power grids," IEEE Systems Journal, vol. 13, no. 1, pp. 625-634, 2019. 
[17] S. Hasan, N. Gurung, K. M. Muttaqi, and S. Kamalasadan, "Electromagnetic field-based control of distributed generator units to mitigate motor starting voltage dips in power grids," IEEE Transactions on Applied Superconductivity, vol. 29, no. 2, pp. 1-4, 2019.

[18] A. B. Birchfield, T. J. Overbye, and K. R. Davis, "Educational applications of large synthetic power grids," IEEE Transactions on Power Systems, vol. 34, no. 1, pp. 765-772, 2019.

[19] S. Bhattacharya and S. Mishra, "Efficient power sharing approach for photovoltaic generation based microgrids," IET Renewable Power Generation, vol. 10, no. 7, pp. 973-987, 2016.

[20] A. Marini, M. S. Ghazizadeh, S. S. Mortazavi, and L. Piegari, “A harmonic power market framework for compensation management of DER based active power filters in microgrids," International Journal of Electrical Power \& Energy Systems, vol. 113, no. 12, pp. 916-931, 2019.

[21] Y. Seyedi, H. Karimi, and S. Grijalva, "Irregularity detection in output power of distributed energy resources using PMU data analytics in smart grids," IEEE Transactions on Industrial Informatics, vol. 15, no. 4, pp. 2222-2232, 2019.

[22] H. H. Cho and V. Strezov, "Comparative analysis of the environmental impacts of Australian thermal power stations using direct emission data and GIS integrated methods," Energy, vol. 231, no. 9, article 120898, 2021.

[23] L. Kai, P. Zaixing, W. Song, and G. Liu, "Research on GIS internal overheating engineering simulation technology," High Voltage Apparatus, vol. 56, no. 6, p. 7, 2020.

[24] M. M. Damoom, S. Hashim, M. S. Aljohani, M. A. Saleh, and N. Xoubi, "Potential areas for nuclear power plants siting in Saudi Arabia: GIS-based multi-criteria decision making analysis," Progress in Nuclear Energy, vol. 110, no. 1, pp. 110-120, 2019.

[25] C. M. Rergis, R. J. Betancourt, E. B. Espejo, and A. R. Messina, "Fourier-series approach to model order reduction and controller interaction analysis of large-scale power system models," IET Generation Transmission \& Distribution, vol. 12, no. 6, pp. 1247-1255, 2018. 